\title{
LA PENSÉE DESIGN COMME APPROCHE PÉDAGOGIQUE AFIN DE CONCEVOIR DES SOLUTIONS À UNE PROBLÉMATIQUE DE QUALITÉ DE L'EAU
}

\author{
Anne-Marie Laroche, Michel T. Léger, Diane Pruneau \\ Université de Moncton \\ anne-marie.laroche@umoncton.ca,michel.leger@umoncton.ca,diane.pruneau@umoncton.ca
}

\begin{abstract}
Résumé - Les ingénieures et les ingénieurs sont amenés à résoudre des problèmes techniques et environnementaux complexes. Ce faisant, elles et ils doivent prendre en compte les impacts de leurs décisions sur les citoyens. Afin de préparer les futures ingénieures et les futurs ingénieurs à ces enjeux difficiles, des étudiants en génie civil ont été exposés à l'approche de la pensée design. Cette dernière est une façon créative et collaborative de résoudre des problèmes. Cet article présente un volet d'une étude exploratoire dont le but est de mieux comprendre leur expérience d'apprentissage en conception. Les données ont été récoltées à l'aide d'entretiens semi-dirigés et d'observations lors des séances de travail en équipe. Les résultats de cette étude montrent que les étudiants ont pris en compte de manière plus explicite les besoins de la population locale. De plus, les recommandations formulées mettent en évidence une multitude de solutions adaptée aux divers besoins des usagers.
\end{abstract}

Mots-clés : Pensée design, Génie civil, Conception, Qualité de l'eau.

\section{INTRODUCTION}

Une ou un ingénieur est une personne qui résout des problèmes techniques, réels et complexes dans le dessein d'améliorer la qualité de vie de ses concitoyens de même que divers systèmes, produits ou services. Ainsi, l'ingénieur contribue, à l'aide de sa formation scientifique et technique, aux mieux-être de la collectivité en concevant des solutions adaptées aux différents problèmes économiques, sociaux et environnementaux. L'objectif de la conception est de créer des systèmes ou des processus qui répondent à un besoin formulé par un client en tenant compte de contraintes [1]. D’après Ingénieurs Canada, la conception se définit comme étant « un processus créatif, itératif et évolutif qui est assujetti à des contraintes pouvant être régies par des normes ou des lois à divers degrés selon la spécialité. Ces contraintes peuvent être liées à des facteurs comme l'économie, la santé, la sécurité, l'environnement et la société ou à d'autres facteurs interdisciplinaires » [2]. La conception conventionnelle en ingénierie se décline en cinq étapes : l'analyse des besoins du client, l'élaboration du concept, la conception préliminaire, la conception détaillée et la synthèse [1]. Cette approche est centrée principalement sur l'utilisation optimale de ressources par le biais d'un cahier des charges. Par ailleurs, il n'est pas rare de constater que les solutions amenées par les étudiants de premier cycle sont souvent technicocentrées. Autrement dit, les futurs ingénieurs ont tendance à négliger d'examiner, d'analyser et de prévoir les impacts des interactions entre les composantes de leur mandat et les aspects liés à l'économie, la santé, la sécurité, les lois, le développement durable de même que la culture de la société. En fait, les solutions proposées dans le cadre de leurs travaux universitaires tiennent compte principalement du client et incluent que rarement la perspective des personnes (usagers) touchées par le projet.

Face à cette problématique, l'exploration d'une nouvelle approche pédagogique positionnant l'aspect social et humain au cœur du processus de conception s'avère pertinente pour outiller les futurs ingénieurs dans la résolution de problèmes complexes. L'adoption de la pensée design comme approche pédagogique semble donc un changement de posture plus approprié aux réalités auxquelles devront faire face les étudiants en génie civil une fois sur le marché de l'emploi. Déjà en 1992, Buchanan associe la pensée design à plusieurs sphères dont «les activités et services organisationnels ainsi que les systèmes complexes ou environnementaux» [8]. Respectant la démarche du modèle conventionnel de conception, la pensée design apparait comme un processus achevé qui permet d'atteindre les connaissances, les pratiques exemplaires ainsi que les compétences requises par le Bureau canadien d'agrément des programmes de génie (BCAPG) [2]. La pensée design émerge au milieu des années 1940. Durant la vingtaine d'années suivante, on assiste à l'établissement des fondements de la pensée design par le raffinement de la technique du remueméninge [3], par une approche scientifique des sciences de la créativité [4], et le rapprochement de la technologie à la 
créativité [5]. Ce n'est qu'en 1984, avec les travaux de Rittel [6] que l'on voit apparaitre dans le processus de conception des approches collaboratives qui incluent une compréhension approfondie du problème. Mais c'est vraiment en 1987 à la Stanford University que les bases de la pensée design sont posées pour réaliser des tâches complexes [7]. Depuis les années 1990, nous assistons à une vision holistique de l'approche de la pensée design dans le processus de conception lié à des problématiques complexes et interdisciplinaires [8] [9] [10] [11].

Cet article traite donc de la pensée design comme approche pédagogique privilégiée dans un cours de traitement des eaux de la formation en génie civil à l'Université de Moncton (Nouveau-Brunswick, Canada). Un des objectifs principaux était d'explorer la manière d'adapter la pensée design et ses outils pour soutenir des étudiants en génie civil dans leur projet de conception afin d'améliorer leur compréhension des interactions de leur solution avec la société et l'environnement. Plus spécifiquement, le volet de cette étude tend à mettre de l'avant le regard des étudiantes et des étudiants lors de l'application systématique des étapes de la pensée design dans le cadre de leur projet universitaire.

Dans un premier temps, la description du projet de recherche est exposée suivie d'une brève présentation du cadre théorique sur lequel repose cette étude. Ensuite, la méthodologie est décrite soit les moyens utilisés pour colliger et traiter les données. Les résultats seront, par la suite, présentés et analysés. Finalement, la conclusion présentera une synthèse des résultats, les limites du projet et des pistes de recherche futures.

\section{DESCRIPTION DU PROJET}

Ce projet de recherche fait partie d'une étude plus large qui a pour but d'identifier et de diffuser des possibilités offertes par la pensée design et les TIC (Technologies de l'Information et de la Communication) en cocréation de solutions à des problèmes environnementaux. Afin de comprendre comment des apprenants ont utilisé la pensée design pour résoudre des problèmes sociétaux, trois sites ont été étudiés pour ces études de cas à savoir Québec, Ottawa et Moncton au cours des années 2018 et 2019. Au site de Moncton, les chercheurs se sont intéressés à l'enseignement de la conception en ingénierie lors de la résolution d'un problème concret de qualité d'eau potable.

Dans le cadre d'un cours de traitement des eaux à l'Université de Moncton, un projet de conception relatif à la distribution de l'eau exempt d'arsenic a été proposé aux étudiants. Ils ont reçu le mandat d'élaborer une solution qui permettra de fournir à des résidents d'une communauté du Nouveau-Brunswick une eau saine et sécuritaire conforme aux normes de Santé Canada. Pour ce faire, ils devaient considérer, entre autres, les caractéristiques d'utilisation de l'eau de cette population, le financement public disponible et les prévisions de développements de la municipalité.
Pour réaliser le projet, les étudiants devaient utiliser les principes de la pensée design. Cette dernière est une façon créative et collaborative de travailler durant laquelle l'intuition importe beaucoup, les solutions sont nombreuses, l'expérimentation arrive rapidement, les échecs sont perçus comme des apprentissages et, surtout, les besoins des usagers, dans ce cas-ci les citoyens de la région, sont pris en compte. Elle se déroule selon des étapes définies comme suit :

\section{1. Éprouver de l'empathie (Observation-} inspiration): en tant que concepteur centré sur l'être humain, il est essentiel de bien comprendre les particularités des usagers. Habituellement, une étude descriptive des usagers est effectuée pour comprendre les personnes concernées par le problème et la situation. On suit les personnes dans leur vie quotidienne pour saisir leurs aspirations et leurs besoins non satisfaits. Les problèmes à résoudre sont rarement les mêmes que celui du concepteur. Il est primordial de développer une empathie envers les usagers et ce qui est important pour eux.

À cette étape, les étudiantes et étudiants se sont, dans un premier temps, approprié la notion d'empathie, d'observation/inspiration. Pour ce faire, elles et ils ont dû définir ensemble qui pouvait être les usagers, les utilisateurs ou les personnes d'intérêts liées au projet. Par la suite, elles et ils ont dû réfléchir à la manière dont elles et ils allaient s'y prendre pour déterminer les besoins des divers usagers.

2. Définir (Synthèse du problème) : cette étape consiste à classer et à synthétiser les résultats obtenus lors de l'étape d'observation-inspiration. Les deux objectifs de cette étape sont d'accroitre une compréhension profonde des utilisateurs et de formuler un énoncé de problème pouvant donner lieu à une action. Habituellement, le problème est défini à plusieurs reprises et de diverses façons. Diverses perspectives du problème sont énoncées. Par la suite, l'information est synthétisée pour poser le problème en quelques énoncés.

Lors de cette étape, les étudiantes et étudiants ont dû regrouper toutes les informations, les classer et ensuite fournir une définition du problème à résoudre.

3. Concevoir (Idéation) : le but de l'idéation est d'explorer de nombreuses idées diversifiées.

Les étudiantes et étudiants ont dû générer un ensemble d'idées novatrices afin de pouvoir résoudre le problème défini précédemment. Pour ce faire, elles et ils devaient aussi schématiser, à l'aide de dessins, la conception retenue.

4. Créer un prototype (Prototypage-essais) : l'objectif ici est de construire des prototypes illustrant les idées proposées à l'étape d'idéation dans le but de les 
partager avec d'autres et d'évaluer leur potentiel. L'évaluation des prototypes se fait en allant chercher les opinions d'experts, de novices et d'utilisateurs.

Lors de cette étape, le prototype a seulement été conçu sur papier, c'est-à-dire dessiner. Les usagers n'ont pas eu la chance de manipuler le prototype comme tel.

5. Communiquer (Communication) : l'objectif est de diffuser les résultats du projet afin de les faire connaitre.

À cette étape, les différentes équipes ont communiqué leur travail à l'aide d'un rapport écrit.

La pensée design est un processus précis, itératif et systématique qui s'emploie à comprendre les buts, le vécu et les contraintes des usagers, à définir les paramètres techniques et stratégiques d'un projet de conception et à dessiner et à imaginer des solutions.

\section{CADRE THÉORIQUE}

La pensée design est une approche itérative, centrée sur les besoins des usagers, tout en étant concrète et flexible quant aux essais et erreurs. L'approche mise sur l'empathie et l'optimisme des utilisateurs. Elle est à la fois inductive, déductive et abductive.

La pensée design n'est pas un processus linéaire puisque le concepteur navigue entre le problème et la solution. Comparativement à une approche conventionnelle de conception, la pensée design se concentre itérativement sur le problème et les solutions potentielles. La définition du problème ne se penche pas uniquement sur une problématique technique, mais accorde une importance aux usagers selon leur vécu, leur point de vue et leur situation. Les concepteurs s'engagent à analyser le problème et les comportements des utilisateurs dans leur environnement. Il est impératif pour les concepteurs d'approfondir et d'acquérir des connaissances à propos du problème. Les solutions, quant à elles, proviennent de pistes multiples réalisées à partir de plans et de prototypes. Ils servent de base de discussion et d'apprentissages.

Lorsque l'on parle de l'usager ou de l'utilisateur, nous faisons référence à toute personne qui pourrait être affectée négativement ou qui pourrait bénéficier de la solution. L'objectif ultime est de trouver une solution qui réponde aux besoins de ces personnes et qui améliore leur niveau de vie. Pour ce faire, tout au long du processus, il faut constamment impliquer l'usager afin de connaitre ses remarques et commentaires en vue d'améliorer la solution. Souvent, ces dernières sont nombreuses, créatives et assurent le succès du projet.

Rapidement, les idées et les solutions se dessinent à l'aide de prototypes sous forme schématique, physique ou numérique. Le but est de constamment revoir la proposition de solution afin de répondre aux besoins des utilisateurs. Par les essais et les erreurs, processus d'apprentissage du concepteur, la solution se raffine.
La pensée design fait appel au raisonnement déductif, inductif et abductif. Les ingénieurs sont habitués à faire appel au raisonnement déductif (général au particulier) et au raisonnement inductif (particulier au général) dans le processus de conception. Toutefois, ils font rarement appel à la pensée abductive. Cette dernière consiste à choisir des hypothèses les plus vraisemblables pour expliquer un phénomène dans le but de tirer des conclusions probables, mais pas certaines qui concordent à des observations. Dans le processus de pensée design, les hypothèses vraisemblables sont formulées par les usagers ou les utilisateurs. Ceci amène donc les ingénieurs à revoir leur processus de conception en tenant compte de leurs observations et raisonnement technique tout en intégrant ceux des usagers.

\section{MÉTHODOLOGIE}

Cette étude est basée sur une méthode de recherche qualitative, de nature exploratoire, à l'aide d'une étude de cas [12]. Nous avons étudié le cas d'étudiants en génie civil utilisant la pensée design comme outil de conception à un problème environnemental.

L'étude a suivi des étudiants inscrits dans un cours de traitement des eaux, d'un programme de génie civil de l'Université de Moncton (Nouveau-Brunswick) au cours de leur semestre d'hiver de 2019. Ils ont dû concevoir une solution afin de résoudre une problématique de qualité d'eau potable dans une communauté rurale où la concentration en arsenic dépasse les normes acceptables. La classe de 17 étudiants a été divisée au hasard en deux groupes distincts, le premier, le groupe contrôle, utilisant l'approche conventionnelle de conception $(n=8)$ et le deuxième, le groupe à l'étude utilisant l'approche de pensée design $(n=9)$. Dans une recherche qualitative, la qualité des données recueillies détermine le nombre de participants à une étude. Dans le cas qui nous intéresse ici, aucun échantillonnage n'était nécessaire, car il n'y avait qu'une seule classe en traitement des eaux et l'intégralité des étudiants inscrits $(\mathrm{n}=17)$ a participé à l'étude. En réalisant des entretiens collectifs avec l'un des deux sousgroupes d'étude plus petits, la méthodologie est conforme aux recommandations selon lesquelles un entretien avec un groupe de discussion devrait comprendre entre six et douze participants [13] [14]. Dans cet article, seulement les résultats obtenus par le groupe de pensée design seront présentés.

Les données ont été collectées durant les quatre mois de la session d'hiver 2019, grâce à des entretiens de groupe semi-dirigés à chaque étape de conception. La professeure et le chercheur ont également tenu un journal, dans lequel des observations et des réflexions analytiques continues ont été enregistrées pour chaque groupe pendant toute la période d'expérimentation. Enfin, un assistant de recherche était présent à toutes les étapes, pour les deux groupes, et a enregistré des observations spécifiques par rapport aux 
compétences développées par les étudiants lors du processus de conception. En collectant et en analysant des données provenant de sources multiples (c'est-à-dire divers outils ou techniques de collecte), la fiabilité des résultats de l'étude est améliorée par triangulation [15] [12].

Une analyse de contenu thématique a été appliquée aux entretiens transcrits afin de décoder les réponses des participants et de construire une image des perspectives et des stratégies utilisées lors du processus de conception respectif par les deux groupes. Les observations colligées dans les journaux des chercheurs ont servi de données de triangulation. L'analyse thématique est un processus analytique qualitatif par lequel des modèles ou des thèmes sont identifiés dans les données qualitatives. Plus spécifiquement, le chercheur développe des codes, des mots ou des phrases qui servent d'étiquettes à des sections de données, menant à terme à l'identification de thèmes émergents [16].

Un questionnaire d'entrevue semi-structuré était composé de cinq questions, qui ont servi à guider les discussions de groupe, sans orienter strictement les réponses. En posant les cinq mêmes questions à chaque entretien (il y a eu un entretien séparé à chacune des étapes de la conception pour les deux groupes), un tableau comparatif complet des expériences des étudiants participants a été établi en utilisant leur approche respective de conception. Voici les questions posées à chaque étape de l'étude :

1. Que retenez-vous de cette étape?

2. Qu'avez-vous appris sur vous?

3. Quelles sont les difficultés rencontrées?

4. Quels sont les points positifs de cette étape?

5. Quelles compétences ont été mobilisées par vous-mêmes ou par le groupe?

\section{RÉSULTATS ET DISCUSSION}

Les résultats sont présentés aux tableaux 1 à 4 selon les deux aspects les plus importants qui sont ressortis, à savoir, le processus de conception et les éléments ressortis lors des entrevues semi-dirigés et des observations notées à partir du journal des chercheurs.

Le tableau 1 montre les principaux éléments observés à chacun des questionnements pour la première étape associée à la pensée design, c'est-à-dire l'empathie. Pour les étudiants, cette phase a nécessité beaucoup plus de temps qu'imaginé initialement. En effet, ces derniers ont passé plus de temps à rechercher de l'information due à la nouveauté de cette étape. Cela a nécessité un certain temps d'adaptation de leur part. Malgré tout, les étudiants ont trouvé que le fait de tenir compte des besoins des usagers dès le départ du processus de conception leur a permis d'intégrer ces besoins dans la solution. Pour eux, cet élément est très positif parce qu'ils ont été placés devant une situation nouvelle. Ils ont également souligné que cette étape leur avait permis de prendre conscience de l'Autre, ce qui a été apprécié par certains étudiants. En effet, ils ont indiqué que l'intégration de l'aspect humain dans un processus de conception était appréciable, surtout pour résoudre des problèmes complexes.

Tableau 1: Principaux éléments ressortis associés à l'étape 1 - Empathie (Observation-Inspiration).

\begin{tabular}{|l|c|}
\hline \multicolumn{1}{|c|}{ Questionnement } & Éléments observés \\
\hline Ce qui a été retenu & $\begin{array}{c}\text { Investissement de temps } \\
\text { plus grand au départ }\end{array}$ \\
\hline Ce qui a été appris & $\begin{array}{c}\text { Ouverture aux autres } \\
\text { Adaptation aux nouvelles } \\
\text { situations }\end{array}$ \\
\hline $\begin{array}{l}\text { Ce qui a causé des } \\
\text { difficultés }\end{array}$ & Défi organisationnel \\
\hline Ce qui a été positif & $\begin{array}{c}\text { Prise de décisions à partir } \\
\text { des besoins des usagers }\end{array}$ \\
\hline $\begin{array}{l}\text { Quelles compétences } \\
\text { ont été mobilisées }\end{array}$ & $\begin{array}{c}\text { Communication } \\
\text { interpersonnelle }\end{array}$ \\
\hline
\end{tabular}

Au tableau 2, nous remarquons que les étudiants ont trouvé qu'ils ont eu plus de facilité à définir le problème. Ils n'ont pas été déstabilisés par cette étape; ce qui n'est pas vraiment surprenant, car la définition du problème fait partie des apprentissages dès les premières années de la formation en ingénierie.

Nonobstant cette facilitation de la réalisation de cette étape, les étudiants ont indiqué qu'il leur a fallu déployer plus de compétences organisationnelles, surtout en ce qui a trait aux données et aux informations recueillies. Cela leur a demandé de filtrer, de trier et de synthétiser le matériel récolté lors de la première étape, un travail intellectuel qui leur a paru ardu par moment. Cela étant dit, les rencontres avec les usagers leur ont permis de mieux cibler la problématique et d'améliorer leur compréhension des enjeux locaux.

Tableau 2: Principaux éléments ressortis associés à l'étape 2 - Définir (Synthèse du problème).

\begin{tabular}{|l|c|}
\hline \multicolumn{1}{|c|}{ Questionnement } & Éléments observés \\
\hline Ce qui a été retenu & $\begin{array}{c}\text { Importance de la rencontre } \\
\text { avec les usagers }\end{array}$ \\
\hline $\begin{array}{l}\text { Ce qui a été appris } \\
\text { Ce qui a causé des } \\
\text { difficultés }\end{array}$ & Tri des informations \\
\hline
\end{tabular}




\begin{tabular}{|l|c|}
\hline Ce qui a été positif & $\begin{array}{c}\text { Facilitation à définir le } \\
\text { problème }\end{array}$ \\
\hline $\begin{array}{l}\text { Quelles compétences } \\
\text { ont été mobilisées }\end{array}$ & $\begin{array}{l}\text { Communication } \\
\text { interpersonnelle }\end{array}$ \\
\hline
\end{tabular}

Malgré ces difficultés, certains ont mentionné que cette étape leur a semblé plus facile à réaliser que la précédente, n'étant pas nouvelle pour eux.

L'étape de conception (tableau 3) montre que les étudiants ont mobilisé plusieurs compétences, à savoir l'imagination, l'innovation, l'optimisme et la persévérance. Ils ont aussi mentionné qu'ils ont pu générer plusieurs idées différentes et variées en lien avec les besoins des usagers. Sur cet aspect, ils ont précisé que, malgré la difficulté de tenir compte des besoins des usagers, cela leur avait permis d'être plus imaginatifs. Ce qui a été perçu comme positif pour eux. Ils ont également exprimé que les solutions qu'ils ont trouvées leur semblaient meilleures, par le fait qu'elles répondaient mieux à la problématique des usagers.

D'ailleurs, ils ont précisé que la pensée design leur a permis de formuler des solutions intégrant des éléments non techniques. Pour eux, cela s'avère un avantage par rapport à la méthode de conception dite «standard». En effet, ils ont mentionné qu'ils ont déployé plus d'imagination dans le processus de conception et, selon eux, qu'il faudrait faire plus de place à la créativité dans leur formation universitaire.

Tableau 3: Principaux éléments ressortis associés à l'étape 3 - Concevoir (Idéation).

\begin{tabular}{|l|c|}
\hline Questionnement & Éléments observés \\
\hline Ce qui a été retenu & $\begin{array}{c}\text { Respect des idées des } \\
\text { usagers }\end{array}$ \\
\hline Ce qui a été appris & Génération de plus d'idées \\
\hline $\begin{array}{l}\text { Ce qui a causé des } \\
\text { difficultés }\end{array}$ & $\begin{array}{c}\text { Difficultés à trouver des } \\
\text { solutions respectant les } \\
\text { besoins des usagers }\end{array}$ \\
\hline Ce qui a été positif & $\begin{array}{c}\text { Élaboration d'une plus } \\
\text { grande diversité de } \\
\text { solutions }\end{array}$ \\
\hline $\begin{array}{c}\text { Queléativité } \\
\text { Innovation } \\
\text { ont été mobilisées } \\
\text { Optimisme } \\
\text { Persévérance }\end{array}$ \\
\hline
\end{tabular}

Tableau 4: Principaux éléments ressortis associés à l'étape 4 - Créer un prototype (Prototypage-essais).

\begin{tabular}{|l|c|}
\hline \multicolumn{1}{|c|}{ Questionnement } & Éléments observés \\
\hline Ce qui a été retenu & $\begin{array}{c}\text { Importance de vulgariser la } \\
\text { solution auprès des usagers }\end{array}$ \\
\hline Ce qui a été appris & $\begin{array}{c}\text { Étape sans élément } \\
\text { nouveau }\end{array}$ \\
\hline $\begin{array}{l}\text { Ce qui a causé des } \\
\text { difficultés }\end{array}$ & $\begin{array}{c}\text { Difficultés à vulgariser } \\
\text { Ce qui a été positif }\end{array}$ \\
\hline $\begin{array}{c}\text { Contribution des usagers à } \\
\text { la solution }\end{array}$ \\
\hline $\begin{array}{l}\text { Quelles compétences mobilisées } \\
\text { Communication orale et } \\
\text { écrite }\end{array}$ \\
\hline
\end{tabular}

À l'étape du prototypage (tableau 4), les étudiants ont mentionné l'importance de pouvoir vulgariser adéquatement aux usagers la solution proposée. Cet élément est positif pour les étudiants, car ils ont précisé avoir ressenti un sentiment d'accomplissement de leur travail plus grand que lors de projet similaire en conception. En effet, la contribution des usagers au développement de la solution leur a semblé assurer une certaine proximité entre leur travail d'ingénieur et les besoins que les usagers avaient indiqués en début de projet à l'étape 1 .

Ils ont aussi mentionné que ce processus nécessite des itérations dans le sens que si la solution n'est pas adéquate aux yeux des usagers, il faut alors retourner « à la table à dessin » afin de l'améliorer. Ils ont d'ailleurs précisé que de solides compétences en communication orale et écrite sont essentielles lors de l'étape du prototypage. Pour eux, il est impératif de pouvoir expliquer de manière compréhensible à tous les usagers la solution proposée, même à ceux ne possédant pas des compétences techniques.

\section{CONCLUSION}

Ce projet est basé sur une méthode de recherche qualitative, de nature exploratoire, à l'aide d'une étude de cas. Cette étude avait pour but d'adapter la pensée design pour accompagner des étudiants en génie civil dans un projet de conception afin de bonifier leur compréhension des interactions de leur solution avec la société et l'environnement. Les étudiants d'un cours de traitement des eaux ont eu à concevoir une solution à une problématique de contamination de l'eau à l'arsenic. Ils devaient utiliser les principes associés à la pensée design.

Le groupe classe a donc été divisé au hasard en deux sous-groupes, le premier, le groupe contrôle a utilisé une 
approche conventionnelle de conception, alors que le deuxième groupe a utilisé l'approche de pensée design. À l'aide d'entretiens semi-dirigés et d'observation à chaque étape de la conception, les données ont été recueillies durant les quatre mois de la session d'hiver 2019. Seulement les résultats associés à la conception par l'approche de la pensée design sont présentés dans cet article.

Les principaux résultats montrent que les étudiants ont, tout d'abord, été déstabilisés par l'approche de la pensée design, par sa nouveauté dans leur parcours universitaire. En effet, la contribution des usagers dans le processus de conception leur a demandé beaucoup plus de temps au début du processus de conception. Par contre, à la fin du projet, les étudiants ont indiqué que la solution apportée à la problématique de contamination de l'eau était meilleure qu'une solution conventionnelle due au fait que les besoins des usagers avaient été pris en compte tout au long du processus de conception.

Les étudiants ont particulièrement mis l'accent sur le fait que l'approche de pensée design leur a permis de développer leur créativité lors de l'élaboration de solutions à des problématiques de qualité de l'eau. Ce dernier aspect leur a semblé essentiel dans leur formation universitaire.

Finalement, l'approche de pensée design a nécessité chez eux de développer leur compétence en communication orale et écrite. Chaque étape du processus nécessitait une interaction avec les usagers. Ils ont réalisé l'importance de pouvoir vulgariser et d'expliquer des phénomènes complexes à des non-initiés de la contamination des eaux.

Malgré le fait que les résultats de cette étude semblent positifs, il faut noter que cette recherche est de nature exploratoire, il est donc nécessaire d'effectuer d'autres études dans ce domaine.

\section{Remerciements}

Nous tenons à remercier les étudiantes et les étudiants du cours de traitement des eaux de la session d'hiver 2019 qui ont accepté de participer à ce projet de recherche. Sans votre franchise, votre analyse et votre dévouement, nous n'aurions pas pu produire tous ces résultats.

Nous tenons également à remercier le Conseil de recherches en sciences humaines (CRSH) pour son appui financier dans ce projet.

Le Comité d'éthique de la recherche avec les êtres humains de l'Université de Moncton a approuvé ce projet [Dossier 1819-024].

Finalement, les auteurs remercient les réviseurs anonymes qui ont permis d'améliorer la qualité du texte.

\section{Références}

[1] Ordre des ingénieurs du Québec. Guide de pratique professionnelle. Montréal, Québec, 2018, 778 pp. \{ISBN 978-2-923766-12-6 (PDF)\}
Disponible en date du 14 février 2021 au : http://oiq.qc.ca/Documents/DSIP/20181024_GPP.pdf

[2] Ingénieurs Canada. Normes et procédures d'agrément. Ottawa, Ontario, 2020, 122 pp. \{ISSN 1708-8054\} https://engineerscanada.ca/sites/default/files/accreditat ion/2021-2022-cycle/accreditation-criteria-procedures2020.pdf

[3] Alex F. Osborn, Wake up your mind - 101 ways to develop creativeness. New York, NY: Schriner, 1952, $277 \mathrm{pp}$.

[4] Richard Buckminster Fuller, World design science decade, 1965-1975. Phase I (1965) Document 3. Comprehensive Thinking. Carbondale, Illinois, 1965.

[5] Herbert A. Simon, Michael Barenfeld, "Informationprocessing analysis of perceptual processes in problem solving," Psychological Review, vol. 76, no. 5, pp. 473483, 1969.

[6] Horst Willhelm Jakob Rittel, "Second-Generation Design Methods," in Developments in Design Methodology, N. Cross (Editor), John Wiley \& Sons, UK, 1984, 10 pp.

[7] Peter G. Rowe, Design Thinking, Cambridge Massachusetts. MIT Press, 1987, 229 pp. \{ISBN: 978$0-262-18122-8\}<$

[8] Richard Buchanan, "Wicked problems in design thinking," Design Issues, vol. 8, no. 8, pp. 5-21, Spring 1992.

[9] Richard Buchanan, "Human dignity and human rights: Thoughts on the principles of human-centered design,' Design Issues, vol. 17, no. 3, pp. 35-39, Summer 2001.

[10] Tom Kelley and Jonhantan Littman, The art of innovation: Lessons in creativity from IDEO. New York, NY: Doubleday. 2001, 320 pp. \{ISBN: 9780385499842\}

[11] Tim Brown, Change by design: How design thinking transforms organizations and inspires innovation. New York, NY: Harper Collins, 2009, 272 pp. \{ISBN: 9780061766084\}

[12] Robert E. Stake, The Art of Case Study Research. Thousand Oaks, CA: SAGE Publications, 1995, 175 pp. $\{$ ISBN: 9780803957671$\}$

[13] Boris Blumberg, Donald R. Cooper, et Pamela S. Schindler, Business Research Methods. London: McGraw-Hill Higher Education, 2008, 780 pp. \{ISBN: 9780077157487\}

[14] Tobias O.Nyumba, Kerrie Wilson, Christina J. Derrick et Nibedita Mukherjee, "The use of focus group discussion methodology: Insights from two decades of application in conservation," Methods in Ecology and Evolution, vol. 9, pp. 20-32, 2018.

[15] Norma K Denzin, et Yvonna S. Lincoln, The SAGE Handbook of Qualitative Research. Thousand Oaks, CA: Sage Publications, 2017 (5e éd.), 992 pp. \{ISBN: 9781483349800\} 
[16] Colette Baribeau, "Analyse des données des entretiens de groupe," Recherches qualitatives, vol. 28, no. 1, pp. 133-148, 2009.

Disponible en date du 16 février 2020 au :

http://www.recherche-

qualitative.qc.ca/documents/files/revue/edition regulie re/numero28(1)/numero complet 28(1).pdf\#page $=13$

6 